\title{
BMC Psychology reviewer acknowledgement 2014
}

Catherine M Rice ${ }^{*}$ and Diana M Marshall

\section{Contributing reviewers}

The editors of BMC Psychology would like to thank all our reviewers who have contributed to the journal in Volume 2 (2014).

\author{
Azimatun Noor Aizuddin \\ Malaysia
}

Katie Alcock

UK

Anna-Karin Andershed

Sweden

Glynne Andrew

UK

Janelle Beadle

USA

Patrizia Bisiacchi

Italy

Michael Karl Boettger

Germany

Samantha Bouwmeester

Netherlands

Philip Boyce

Australia

Doug Brock

USA

Christian Bröer

Netherlands

Brendan Bunting

UK

Noriko Cable

UK

Emanuele Antonio Maria Cappella Italy

\author{
Shana Carpenter \\ USA \\ Thep Chalermchai \\ Thailand \\ John Chaplin \\ Sweden \\ Jimmy Choi \\ USA \\ Mary Chong \\ Singapore
}

Kaj Sparle Christensen

Denmark

Deirdre Conroy

USA

Helen Correia

Australia

Janna Cousijn

Netherlands

Georgina Cox

Australia

James Coyne

USA

Cathy Creswell

UK

Simone Croft

UK

Keith Donohue

USA
Pete Ellis

New Zealand

Monica Eriksson

Sweden

George Georgiou

UK

Rebecca Giallo

Australia

George Giannakopoulos

Greece

Kenneth Gilhooly

UK

Donald Goff

USA

Thalia Goldstein

USA

Michael Gradisar

Australia

Gaia Gragnano

Italy

Roser Granero

Spain

Daniel Gruehn

USA

Gareth Hagger-Johnson

UK

Phillipa Hay

Australia

* Correspondence: Catherine.Rice@biomedcentral.com

BioMed Central, Floor 6, 236 Gray's Inn Road, London WC1X 8HB, UK 
Craig Hedge

UK

Masahiro Heima

USA

Sigurd William Hystad

Norway

Tihomir Ilic

Serbia

Gary Jones

UK

Göran Jutengren

Sweden

Jenny Kelly

Australia

Margaret Kern

USA

Dennis Klass

USA

Judith Knausenberger

Germany

Tina Kretschmer

Netherlands

Anne Katrin Külz

Germany

Joanna C. Lee

USA

Rana Limbo

USA

Wolfgang Linden

Canada

Andrew Littlefield

USA

Arnold Lohaus

Germany

Matthew Macfarlane

Australia

Chantal Martin-Soelch

Switzerland

Barbara Maughan

UK

Stella Mavroveli

UK

Adrian Meule

Germany
Michela Milioni

Italy

Robert Miller

Germany

Øyfrid Larsen Moen

Norway

Ho Sik Moon

Korea, South

Erin Morgan

USA

Naser Morina

Switzerland

Julia Müller

Switzerland

Marcus Munafo

UK

Urs Nater

Germany

Chunping Ni

China

Sam Norton

UK

Jason Oliver

USA

Femi Oyebode

UK

Massimiliano Pastore

Italy

Stephanie Penney

Canada

Laura Petitta

Italy

Monique Pfaltz

Switzerland

Vitoria Piai

USA

Kevin Pile

Australia

Belinda Platt

Germany

Olga Pollatos

Germany

Stephanie Potochnick

USA
Livia Puljak

Croatia

Michael Pullmann

USA

Nalini Ranjit

USA

Julia Rao

USA

Andrew Rasmussen

USA

Rinie Rinie Geenen

Netherlands

Stuart Ritchie

UK

Reuben Robbins

USA

Gabriela Roman

UK

Gennaro Ruggiero

Italy

Amanda Sacker

UK

Samaneh Sadeghi

UK

Yasuhisa Sakurai

Japan

Cristina Scarpazza

UK

Virginia Schmied

Australia

André Schulz

Luxembourg

Beate M. Schulze

Switzerland

Stefania Sette

Italy

Kimberley Smith

Canada

Fruzsina Soltesz

UK

Tobias Stalder

Germany

Piers Steel

Canada 
Lisanne Stone

Netherlands

Alexander Strobel

Germany

Serge Sultan

Canada

Lois Surgenor

New Zealand

Phern-Chern Tor

Singapore

Yannis Tountas

Greece
David Trafimow

USA

Gareth John Treharne

New Zealand

Michael Ullman

USA

Peter H Van Ness

USA

Eleonora Volpato

Italy

Jelte Wicherts

Netherlands
Stefan Wiens

Sweden

Gill Windle

UK

Tim Windsor

Australia

Sharlene Wolchik

USA

Stefano Zago

Italy 